\title{
Performance Evaluation of Zigbee Routing Protocol under Various Conditions using OPNET Modeler
}

\author{
Nahla Abdulrahman Hussain \\ University of Baghdad \\ College of science \\ Computer Science Department
}

\author{
Fuad Ali Yaseen \\ University of Baghdad \\ Computer Center
}

\begin{abstract}
Zigbee, which has the standard IEEE 802.15.4. It is advisable method to build wireless personal area network (WPAN) which demands a low power consumption that can be produced by Zigbee technique. Our paper gives measuring efficiency of Zigbee involving the Physical Layer (PL) and Media Access Control (MAC) sub-layer, which allow a simple interaction between the sensors. We model and simulate two different scenarios, in the first one, we tested the topological characteristics and performance of the IEEE802.15.4 standard in terms of throughput, node to node delay and figure of routers for three network layouts (Star, Mesh and Cluster Tree) using OPNET simulator. The second scenario investigates the self-healing feature on a mesh topology from earlier design until it supports a large number of end points $(>64,000)$ with dynamic routing. The self-healing is done by removing a router from the network during operation, and seeing the end devices find an alternate path to communicate with the coordinator.
\end{abstract}

\section{Keywords}

Zigbee, QoS, node to node delay, Throughput, OPNET, Selfhealing.

\section{INTRODUCTION}

Wireless Personal Area Network (WPAN) and Wireless Local Area Network (WLAN) technologies are growing fast (soaring) with new emerging standards being developed [1]. WLAN technologies have been leading the indoor Internet distribution in education, business and home environments. They are usually deployed as wireless extension of a broadband access to the network. These technologies are based on CSMA/CA medium access with a positive MAC layer acknowledgement and a retransmission mechanism that aids noisy channel propagation condition and eventual undetected collisions. Today, WLAN standard defines high rate data throughputs; such as the IEEE $802.11 \mathrm{~b}$ with a maximum throughput of $11 \mathrm{Mbps}$ and the IEEE $802.11 \mathrm{~g}$ with maximum throughput of 54Mbps. Both IEEE $802.11 \mathrm{~b}$ and $\mathrm{g}$ operate at the $2.4 \mathrm{GHz}$ band. Typically, WLAN devices operate within 100 meters of distance range depending on the surrounding environment. While, the IEEE $802.11 \mathrm{~b}$ utilizes direct sequence spread spectrum (DSSS) using complementary code keying (CCK) modulation, IEEE $802.11 \mathrm{~g}$ is based on the orthogonal frequency division multiplexing (OFDM) modulation technique and the CCK modulation for backward compatibility with $802.11 \mathrm{~b}$. For some time, Bluetooth [2] was the most widely used for short range communication in the proximity of a person.

Recently, Zigbee was introduced as an alternative to Bluetooth for devices with low power consumption requirements and applications of lower bit rates. Although products based on the Bluetooth standard are often capable of operating at greater distances, the targeted operational area is the one around an individual, (e.g. within a 10 meters diameter) [3].

The new short range, low power, low rate wireless networking protocol, Zigbee, complements the high data rate technologies such as WLAN and open the door for many new applications. This standard operates at three bands, the $2.4 \mathrm{GHz}$ band with a maximum rate of $250 \mathrm{kbps}$, the $915 \mathrm{MHz}$ band with a data rate of $40 \mathrm{kbps}$, and the $868 \mathrm{MHz}$ band with a data rate of 20 kbps. While bluetooth devices are better suited for high rate sensor and voice applications, Zigbee is better suited for low rate sensors and devices used for control applications that do not require high data rate but must have long battery life, low user interventions and mobile topology. Some of these applications are in the fields of medicine, home/office automation, military, and many others [4].

In the medicine field, sensors utilizing Zigbee are used for monitoring the heartbeat, blood pressure and the percentage of the cholesterol in the blood. In the field of home automation, Zigbee capable sensors can be used in turning On/Off the AC if the temperature exceeded a certain value, turning On/Off the lights and locking doors. In the military field, sensors running Zigbee can be used to observe and track the movement of the enemy.

Recently, there have been several investigations related to Zigbee. However; there are not enough simulation-based performance evaluations of the new standard. One of the performance evaluation studies that used simulation was presented in [5]. In this study, authors evaluated the suitability of the Zigbee standard. Their main objective was investigating the scalability issue, since patients might need several communicating devices near them. They developed models for low-rate WPAN access protocol and evaluated the performance of these models using OPNET. The authors in [6] examine the performance of an IEEE802.15.4/Zigbee MAC based WBAN operating in different patient monitoring environment. They study the performance of a remote patient monitoring system using an OPNET based simulation model. Authors in [7], present an initial implementation of the Zigbee network layer in NS-2, which will allow further research and development to be conducted in this area. Investigation the performance of OPNET modeler in simulating Zigbee WSNs was done in [8]. According to simulation results concluded that Zigbee protocol gives less end to end delay. Traffic dropped in route while travelling to destination is also very less. It can be seen that steady stream of traffic is sent without disruption.

In this paper we are analyzing three different topologies star mesh and cluster tree. The novelty of the work is in the performance of the parameters can be measured by different simulations. These results will be helpful to configure the Zigbee and to select a suitable topology according to situation. Then the self-healing mechanism upon router failure is tested through simulation by providing a trajectory to the router to move it out of range to trigger self-healing. 
The rest of the paper is organized as follows. Section 2 provides a brief summary of the Zigbee/IEEE 802.15.4 standard which includes the physical and MAC layer specifications. Section 3 discusses the Simulation Models and Topologies, while the results of simulation to the two scenarios will be in section 4 and Section 5 concludes the paper giving the results.

\section{OVERVIEW OF ZIGBEE/IEEE 802.15.4}

Zigbee is used in many devices which implement in communication systems that need very low power such as wireless network standard aimed at spacious development of long life battery devices in wireless applications of controlling and monitoring. Zigbee is employed due to it has low-cost, low-power. The general features and specifications of the physical layer and the MAC layer are defined in this section [9].

\subsection{Zigbee Specifications}

General Zigbee specification can be listed in Table (1) below.

Table 1. General ZigBee Specifications

\begin{tabular}{|l|l|}
\hline & Zigbee 802.15.4 \\
\hline Broadcast domain (meters) & $1-100$ \\
\hline Life of battery (days) & $100-1000$ \\
\hline Network size (no. of nodes) & $>64000$ \\
\hline Throughput (kb/s) & $20-250$ \\
\hline Radio band & $2.4 \mathrm{GHz}$ \\
\hline
\end{tabular}

\subsection{Zigbee Layers}

Zigbee consists of four layers. The top two (Application and Network) layers specifications are provided by the Zigbee Alliance to provide manufacturing standards. The bottom two (Medium Access Control and Physical) layers specifications are provided by the IEEE 802.15.4-2006 standard to ensure coexistence without interference with other wireless protocols such as Wi-Fi.

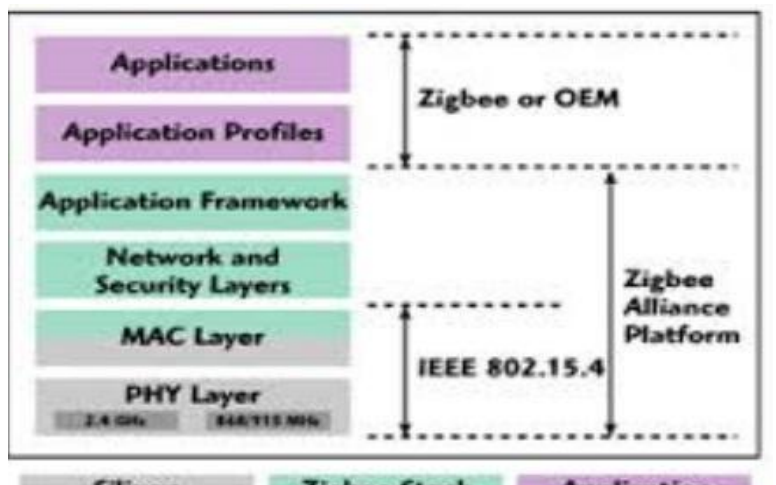

Salicon Zigbeestack Application

\section{Figure (1) Overview of Zigbee Layers [10] \\ 2.2.1 Application Layer}

Applications running on the Zigbee network are contained here. For example, applications to monitor temperature, humidity, or any other desirable atmospheric parameters can be placed on this layer for agricultural use. This is the layer that makes the device useful to the user. A single node can run more than one application. A special application is on every Zigbee device, and this is the Zigbee Device Object, or ZDO. This application provides key functions such as defining the type of Zigbee device (end device, router, and coordinator) a particular node is, initializing the network, and to also participate in forming a network [11].

\subsubsection{Network Layer}

The major tasks of the network layer are to give the correct ability of using the MAC sub layer and providing a proper port to use by the next upper layer (the application layer). Its capabilities and structure are those typically associated to such network layers, including routing. The entity of data is created and processed by network layer data units from the payload of the application layer and performs routing according to the topology which is used [12].

\subsubsection{Medium Access Control Sub-Layer}

This layer extracted from the IEEE 802.15.4 standard provides services to the network layer above, which is part of the Zigbee stack level. The MAC layer is responsible for the addressing of data to determine either where the frame is going, or coming from. It is also this layer that provides multiple access control such as CSMA/CA allowing for reliable transfer of data. Beaconing is another feature implemented through this layer. Finally, the MAC sub-layer can be exploited by higher layers to achieve secure communication [12].

\subsubsection{Physical Layer}

The physical layer considers the nearest layer to the hardware, the controlling and communicating is achieved by it via the radio transceiver directly. It is responsible about all tasks that access to the Zigbee hardware, including starting of the hardware, selecting the channel ,link quality speculation, measuring detected energy and evaluating of clear channel to select the channel. This layer allows for channel selection to avoid radio interference, as well as data exchange with the layer above (MAC sub-layer) to provide it with service [13].

\subsection{Network Devices}

Zigbee networks can contain a mixture of three potential components. These components are a Zigbee coordinator, a Zigbee router, and a Zigbee end device [14].

\section{- Zigbee Coordinator}

This node is responsible for initializing the network, selecting the appropriate channel, and permitting other devices to connect to its network. It can also be responsible for routing traffic in a Zigbee network. In a star topology, the coordinator is at the center of the star, and all traffic from any end device must travel to this node. It is still possible for end devices to talk to another end device, but the message must be routed through the coordinator. In a tree topology, the coordinator is at the top of the tree, and in a mesh network, it is the root node of the mesh. A Zigbee coordinator can also take part in providing security services.

\section{- Zigbee Router}

A router is able to pass on messages in a network, and is also able to have child nodes connect to it, whether it be another router, or an end device. Router functions are only used in a tree or mesh topology, because in a star topology, all traffic is routed through the center node, which is the coordinator. Routers can take place of end devices, but the routing functions would be useless in such cases. If the network supports beaconing, then a router can sleep when inactive, periodically waking up to notify the network of its presence. 


\section{- Zigbee End Device}

The power saving features of a Zigbee network can be mainly credited to the end devices. Because these nodes are not used for routing traffic, they can be sleeping for the majority of the time, expanding battery life of such devices. These nodes carry just enough function to talk to parent nodes, which can be either a router or a coordinator. An end device does not have the ability to have other nodes connect to its network through the end device, as it must be connected to the network through either a router, or directly to the coordinator.

\section{SIMULATION MODELS AND TOPOLOGIES}

Simulation and modeling are important approaches in the development and evaluation of the systems in terms of time and costs. The simulation shows the expected behavior of the system based on its simulation model under different conditions. Hence, the purpose of this simulation model is to determine the exact model and predict the behavior of the real system. For the purpose of simulation, we will use OPNET Modeler 14.5, which is a leading environment for modeling and simulations. This simulation tool provides a comprehensive development environment to support modeling of communication networks and distributed systems, also to better understand the use of OPNET simulation tools to model the protocol of interest, Zigbee.

To achieve these goals, we are considering two scenarios. First, we are comparing the three possible topologies (Star, Mesh and cluster Tree) to each other in terms of throughput, end to end delay and number of hops. While in the second scenario, the feature of the self-healing mechanism in a mesh zigbee network is discussed when one router in the network is failed and who a such network can handle this failure. To provide a brief overview of what zigbee protocol contains, and simulate several simple wireless personal area network (WPAN) while altering certain parameters.

\subsection{First scenario}

In this scenario, Star, Mesh and Cluster Tree topologies in a Zigbee network are considered. The number and type of Zigbee nodes in all three topologies are the same. There is only one ZC (Zigbee Coordinator), six ZR (Zigbee Router) and six ZED (Zigbee End Device). Only one ZR and ZED are mobile, while the others are stationary. The three intended topologies are configured in OPNET according to different simulation parameters of media access control, physical layer, carrier sense multiple access and Application traffic as shown in tables (2), (3) and (4).

Table (2) Purposed Simulation Parameters of Physical Layer

\begin{tabular}{cc}
\hline \multicolumn{2}{c}{ Physical Layer } \\
\hline Data rate & Data rate \\
\hline Receiver Sensitivity & $-85 \mathrm{~dB}$ \\
\hline Transmission Band & $2.4 \mathrm{GHz}$ \\
\hline $\begin{array}{c}\text { Transmission } \\
\text { Power }\end{array}$ & $0.05 \mathrm{~W}$ \\
\hline $\begin{array}{c}\text { MAC (3) Purposed Simulation Parameters } \\
\text { of MAC and CSMA }\end{array}$ \\
\hline \multicolumn{2}{c}{0.05} \\
\hline $\begin{array}{c}\text { ACK wait time } \\
\text { Total }\end{array}$ \\
\hline $\begin{array}{c}\text { Retransmissions } \\
\text { CSMA }\end{array}$ \\
\hline $\begin{array}{c}\text { Exponent of minimum back } \\
\text { off }\end{array}$ \\
\hline $\begin{array}{c}\text { Exponent of maximum back } \\
\text { off }\end{array}$ \\
\hline Carrier sense duration \\
\hline
\end{tabular}

We define two trajectories where the mobile nodes will pass during the simulation progresses. If the mobile node is out of its parent transmission range, then it connects to the closer node and it continuing with the transmissions. The network structure of Star topology is shown on Figure (3). In the Star topology, ZC allows up to 255 child nodes to be connected, and the maximum depth is set to one. We set the Acknowledgment mechanism to "Enable" for every ZED, so every ZED can send an acknowledgment to its parent in order to confirm that it receives the packets.

Table (4) Purposed Application Traffic

\begin{tabular}{|c|c|c|c|c|c|c|}
\hline & \multicolumn{6}{|c|}{ Application Traffic } \\
\hline Parameters & $\begin{array}{c}\text { Device } \\
\text { Type }\end{array}$ & $\begin{array}{c}\text { Inter-arrival } \\
\text { time of Packet }\end{array}$ & Size of Packet & $\begin{array}{c}\text { Start } \\
\text { Time }\end{array}$ & $\begin{array}{c}\text { Stop } \\
\text { Time }\end{array}$ & Destination \\
\hline \multirow{4}{*}{$\begin{array}{c}\text { Star } \\
\text { Topology }\end{array}$} & ZC & Constant (1.0) & Constant (1024) & $\begin{array}{c}\text { Uniform } \\
(20,21)\end{array}$ & Infinity & $\begin{array}{c}\text { All ZCs and } \\
\text { ZRs }\end{array}$ \\
\cline { 2 - 7 } & ZR & Constant (1.0) & Constant (1024) & $\begin{array}{c}\text { Uniform } \\
(20,21)\end{array}$ & Infinity & ZC \\
\cline { 2 - 7 } & ZED & Constant (1.0) & Constant (1024) & $\begin{array}{c}\text { Uniform } \\
(20,21)\end{array}$ & Infinity & ZC \\
\hline \multirow{3}{*}{$\begin{array}{c}\text { Mesh } \\
\text { topology }\end{array}$} & ZC & Constant (1.0) & Constant (1024) & $\begin{array}{c}\text { Uniform } \\
(20,21)\end{array}$ & Infinity & $\begin{array}{c}\text { All ZCs and } \\
\text { ZRs }\end{array}$ \\
\cline { 2 - 7 } & ZR & Constant (1.0) & Constant (1024) & $\begin{array}{c}\text { Uniform } \\
(20,21)\end{array}$ & Infinity & $\begin{array}{c}\text { All ZCs and } \\
\text { ZRs }\end{array}$ \\
\hline & ZC & Constant (1.0) & Constant (1024) & $\begin{array}{c}\text { Uniform } \\
(20,21)\end{array}$ & Infinity & Parents \\
\hline & & Constant (1024) & Uniform & Infinity & ZCs and \\
\hline
\end{tabular}




\begin{tabular}{|c|c|c|c|c|c|c|}
\hline \multirow{4}{*}{$\begin{array}{c}\text { Cluster- } \\
\text { Topology }\end{array}$} & ZR & Constant (1.0) & Constant (1024) & $\begin{array}{c}\text { Uniform } \\
(20,21)\end{array}$ & Infinity & $\begin{array}{c}\text { All ZCs and } \\
\text { ZRs }\end{array}$ \\
\cline { 2 - 6 } & ZED & Constant (1.0) & Constant (1024) & $\begin{array}{c}\text { Uniform } \\
(20,21)\end{array}$ & Infinity & ZRs \\
\hline
\end{tabular}

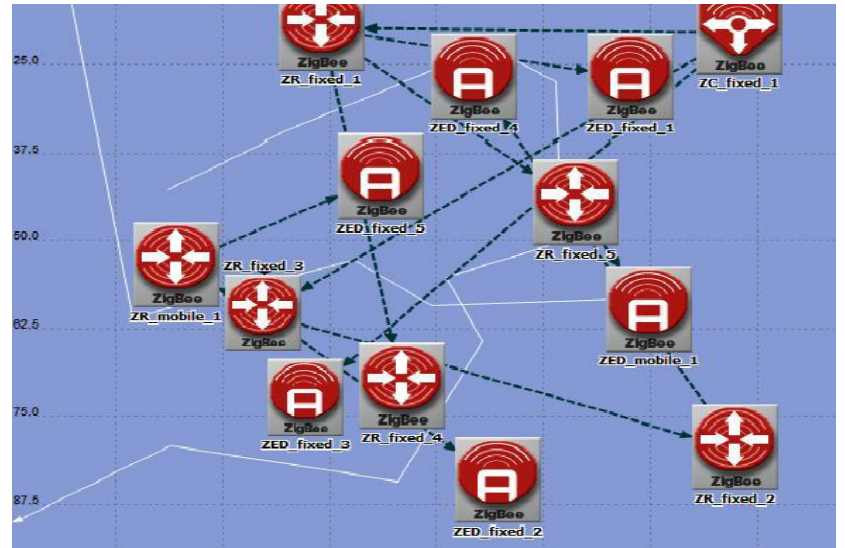

Figure (3) Proposed Star Topology

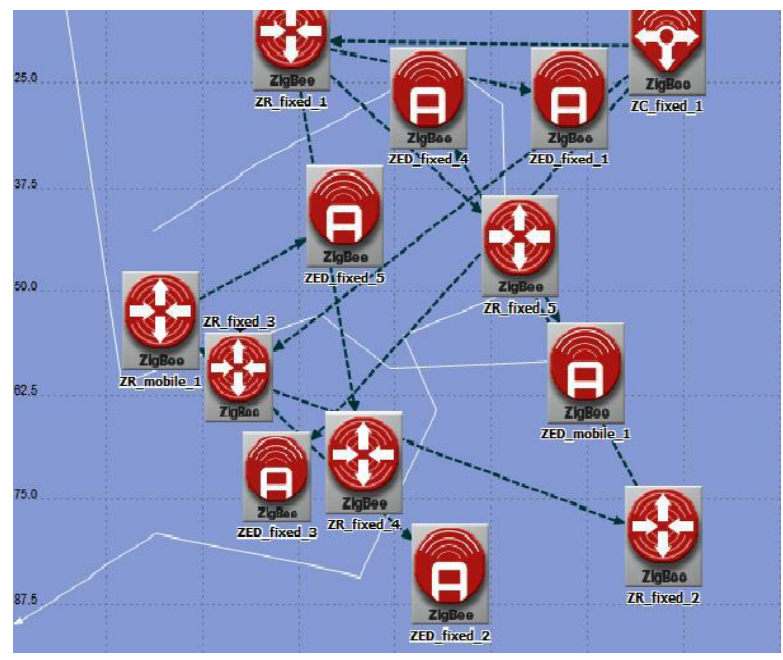

Figure (4) Tree topology

The network structure of Cluster Tree and Mesh topologies are shown on Figure (4) and Figure (5) respectively. The Mesh and the Cluster Tree topologies form the same network structure with the identical seed. The only difference between them is that the mesh topology computes routing table.

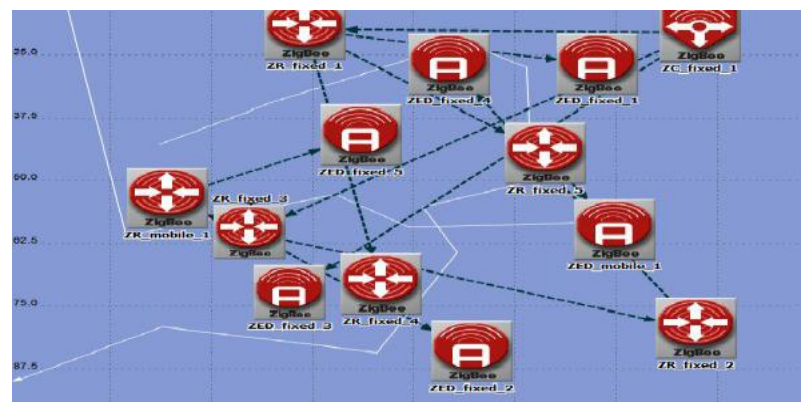

Figure (5) Mesh topology

\subsection{Second scenario}

In this scenario the verification of Zigbee's Self-Healing Mechanism upon Router Failure is simulated. This is done by providing a trajectory to the router to move it out of range to trigger self-healing. This can be analogous to a case of router being blown away in the agricultural application due to extreme winds.

Two key features required for this case scenarios are the ACK enable and understanding the range capability of Zigbee. Placing the end devices too close to the destination coordinator will result in traffic being sent directly, rather than through the router, preventing observations for the self-healing feature. Also the ACK enable was required for the end devices to recognize that the failure in the router has occurred, no longer receiving and routing traffic, in order to trigger route discovery. Figure (6) below illustrates the traffic path from end devices to the coordinator prior to the failure, where Figure (7) illustrates the traffic path after the failure in the bottom router, triggering the self-healing to find an alternate path to the destination.

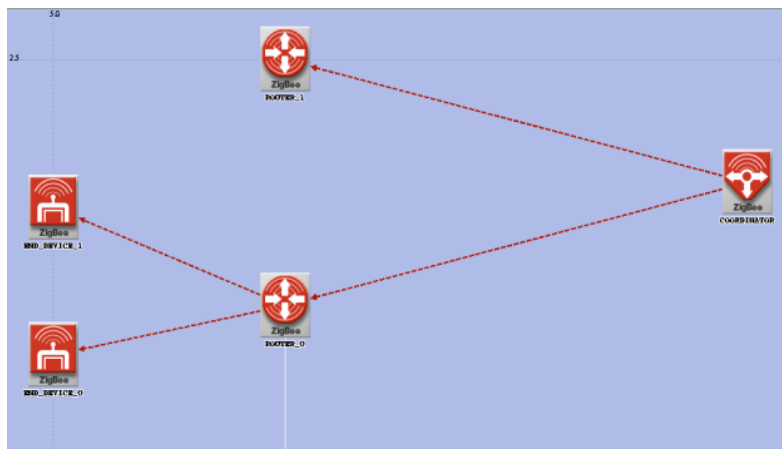

Figure (6) Traffic path prior router failure

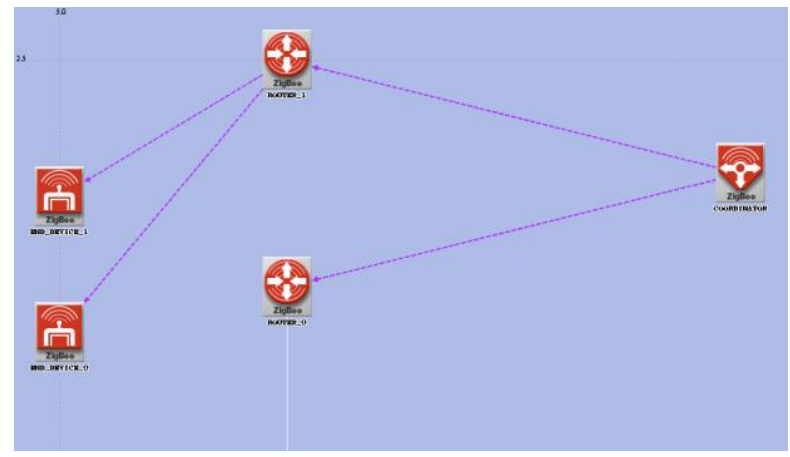

Figure (7) Traffic path after router failure

\section{SIMULATION RESULTS}

The focus of the study of the first scenario is on the quality of service statistics such as delay, throughput and number of hops for the three topologies. Figure (8) shows the node-tonode delay result of the three topologies. The Star and Mesh topologies have similar node-to node delay in this simulation. The node-to-node delay of the cluster tree topology is higher for more than $50 \%$ compared with other two topologies. 
Figure (9) dislpays that the highest throughput is carried out in Cluster Tree topology, the Star topology has second highest throughput while the lowest throughput is for the Mesh topology. The reason for this cases are Tree topology communicates on the basis of the PAN coordinators and ZR which is more efficient as compared to the end devices. Also in Tree topology total load of the network is divided among the local PAN and ZRs as a result of which lesser collisions and lesser packet drops occurs as a result of which the throughput is maximum in case of Tree topology.

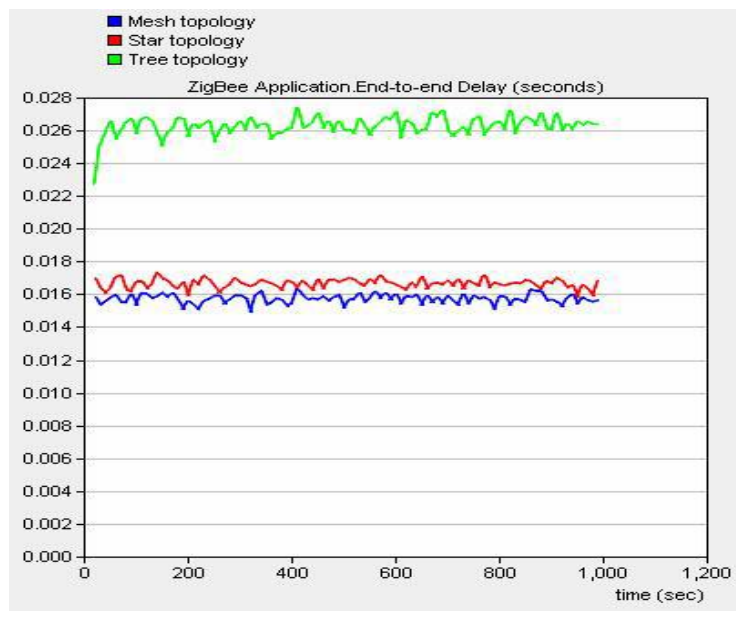

Figure (8) Node-to-Node delay (Star, Tree and Mesh)

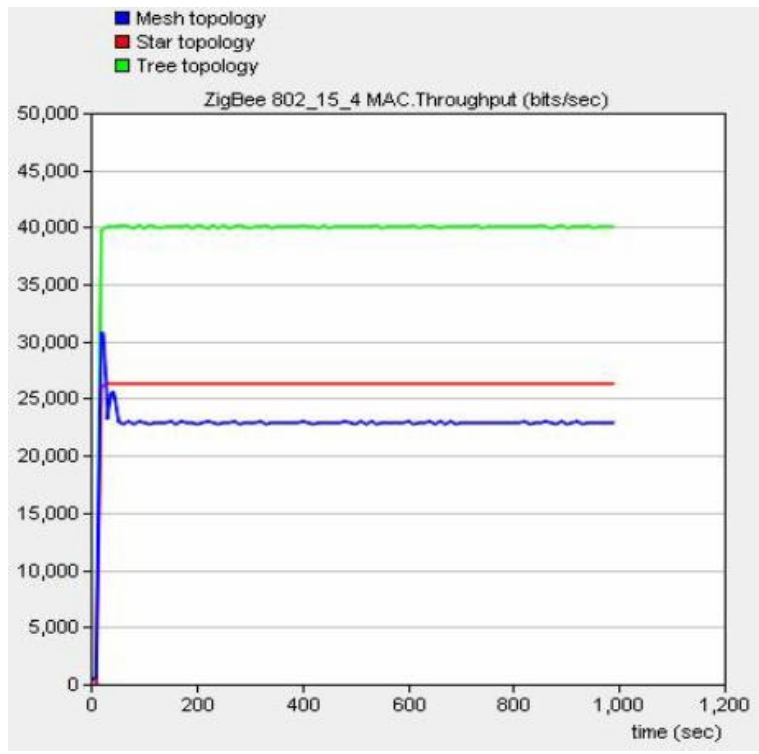

Figure (9) Throughput

The packets travelling through a number intermediate nodes to reach the destination is known as the number of hops. Figure (10) illustrate that the number of hops for Star topology is equal to two, that means the source and the random destinations is separated by another intermediate node, which moves the data. That node in this topology is the coordinator. In Tree topology The number of hops is varied from one to four. Therefore, the network structure depth is three for the simulation, it takes four hops to transport the packet to the further node as a maximum number. The Mesh topology uses a routing table and the average number of hops for simulated scenario is two.

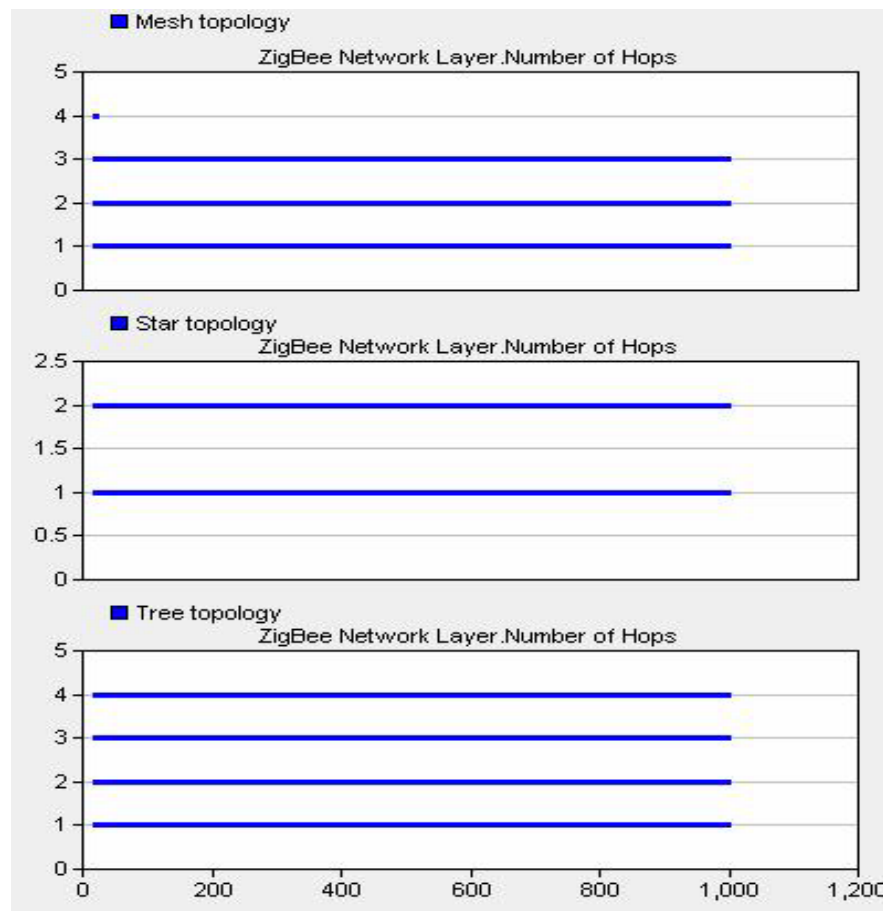

\section{Second Scenario}

Figure (10) Number of hops

On the second scenario, the statistics collected to observe the behavior of self-healing are shown in the figures from (11) to (15). Figure (11) shows the traffic sent by the two end devices (blue line overlapping with red) and traffic received by the two routers. The green line shows a sharp drop at five minutes due to it being moved out of range of the end devices and stops receiving traffic. The light blue line along the top is the stationary router. It shows that router is receiving traffic from all neighboring devices initially. This is due to the lack of a beaconing feature of Zigbee in this model, where non-active devices are able to go into sleep mode, occasionally waking up to notify its presence to the network. Despite the heavy traffic received by the stationary router, it does not transmit (route the traffic) to the destination coordinator as it will be described in the figure (12) and (13).

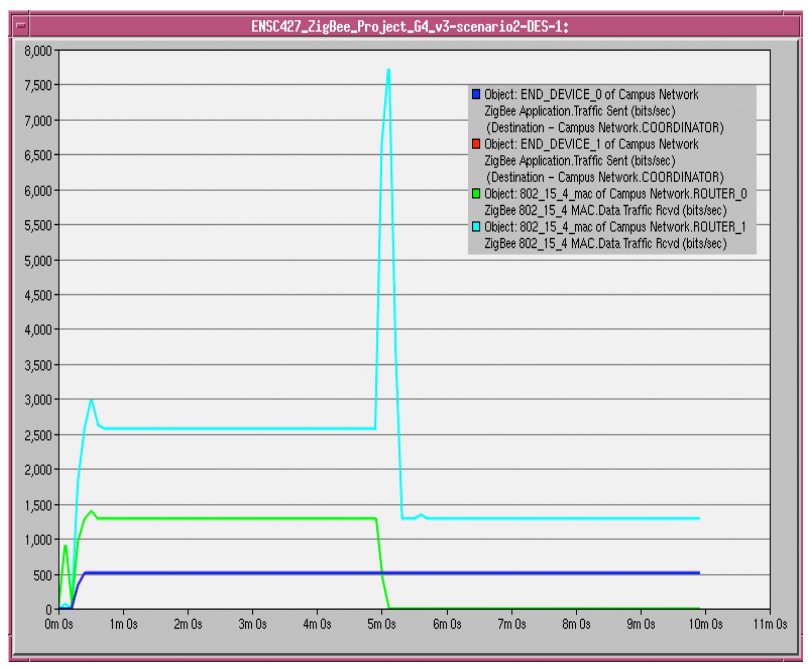

Figure (11) Traffic Sent by end devices and received by routers 
Figures (12) and (13) show the traffic between the two routers and the coordinator (destination). The blue line shows the traffic received by the coordinator while the red and green shows the traffic sent by the routers (to the coordinator). In the first five minutes, it can be seen that stationary router does not send (route) any traffic despite receiving large amounts. The coordinator continuously receives the traffic with one instance of a gap at the five minute simulated time. This is when the self-healing route discovery occurs. The mobile router attempts to find its place in the network (red spike). Once the initial router fails, the stationary router picks up the traffic and routes it to the destination.

The sharp spike near the five minute time is similar to the spike observed near the start of simulation. The spike occurred due to the management control traffic was transmitted by the devices to carry out route discovery. The spike at the five minute is caused by the self-healing feature of Zigbee, it simply recognizes absence of the original path and performs route discovery once again to find the next optimal path to its destination.

Figures (13) and (14) show the node-to-node delay seen from the end devices to the coordinator. This is a measure of time from generating the application packet to the time received by the destination. Figure (13) shows the "as is" node-to-node where the small gap at the five minute simulated time shows packets dropped while the router failure occurred. However, the average node-to-node delay is constant throughout as seen in Figure (14) demonstrating the consistency maintained in network traffic despite a router failure.

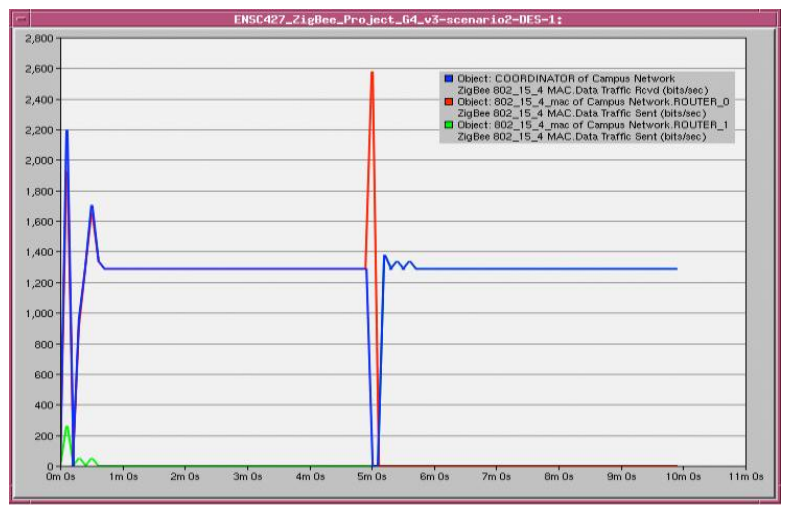

Figure (12) Traffic from routers to coordinator

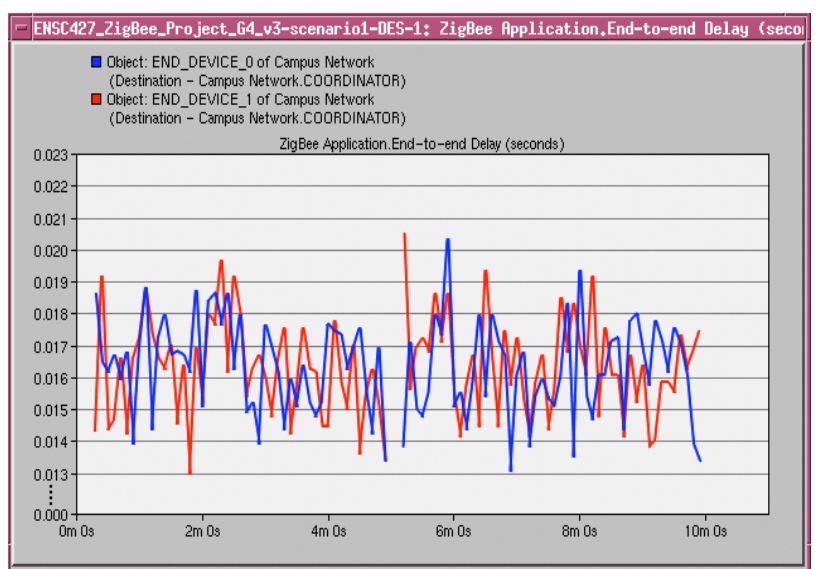

Figure (13) Node to node delay from end devices to coordinator (As Is)

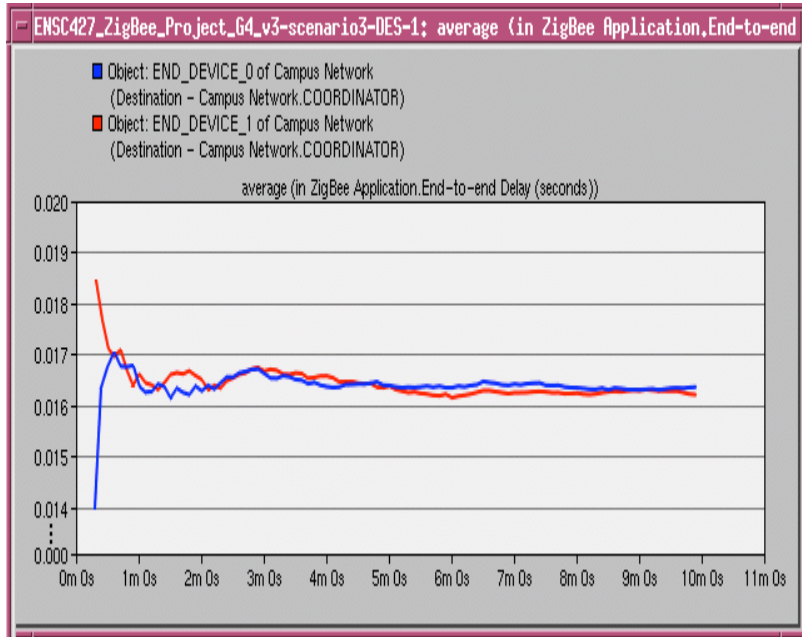

Figure (14) Node to node delay from end devices to coordinator (Average)

\section{CONCLUSION}

In this paper, we presented an overview of the QoS performances of the Zigbee protocol when it applied on wireless sensor networks. The IEEE 802.15.4/ Zigbee protocol stack provides a practical application solution for low data rate, low cost, low energy consumption, and characteristics WSNs. This project focuses on simulation an IEEE 802.15.4/ Zigbee protocol using OPNET simulator.

To test topological characteristics of WSNs, we simulated and analyzed two scenarios. In first scenario, we compared the three possible topologies (Star, Mesh and Cluster Tree) to each other and considered the statistics for node-to-node delay, global throughput and number of hops. In the second scenario, we simulated a failure in the router through providing a trajectory to the router to move it out of range to trigger self-healing. From the results it can be seen that stationary router picks up the end device traffic and continues to route the traffic to the destination.

\section{REFERENCES}

[1] R. Daidone " Experimental evaluations of security impact on IEEE 802.15.4 networks," IEEE International Symposium on a World of Wireless, Mobile and Multimedia Networks, 2011.

[2] Chatschik Bisdikian "An Overview of the Bluetooth wireless technology," IBM Corporation , 2009.

[3] Wu Ling-Xi, Zhan Jie "Access Probability Analysis of IEEE802.15.4," International Conference on Wireless Communications, Networking and Mobile Computing (IEEE WiCOM), pages 475-475, 2007.

[4] Tanabe, I. Sasaki, H. Li Zheng "The implementation of Ultra Low Power IEEE802.15.4 Module and its Application for Battery less Wireless Sensors," IEEE $5^{\text {th }}$ International Conference on Networked Sensing System," pages 93-96, 2008.

[5] N. Golmie, D. Cypher, O. Rebala, "Performance Evaluation of Low Rate WPANs for Sensors and Medical Applications," Proceeding of MILCOM 2004, Monterey, CA, USA October 31-November 3, 2004.

[6] Khan, Jami Y. Mahmet R. Karami "Performance Evaluation of a wireless body area sensor network patient monitoring," $30^{\text {th }}$ Annual International Conference of the IEEE Engineering in Medicine and Biology Society," page 1266-1269, 2008. 
[7] Ling-Jyh Chen, Tony Sun, and Nia-Chiang Liang, " An Evaluation Study of Mobility Support in Zigbee Networks," Proceedings of the 2007 IFIP International Conference on Embedded and Ubiquitous Computing , springer, April 2010, Volume 59, Issue 1, pp 111-122.

[8] Rana Mahajan, Sudha Nair, " Performance Evaluation of Zigbee Protocol Using Opnet Modeler for Mine Safety," International Journal of Computer Science and Network, Vol 2, Issue 1, 2013.

[9] Howitt, I. and Gutierrez, J.A. "IEEE 802.15.4 low rate wireless personal area network coexistence issues", Wireless Communications and Networking, 2003. WCNC 2003. IEEE, Volume: 3, 16-20 March 2003.

[10] http://zigbee layers.
[11] Abhishek Kumar, Sandeep Gupta," International Journal of ENGINEERING Science \& Research Technology," p2733-2738, October 2013.

[12] Lamia Chaari and Lotfi Kamoun," Performance Analysis of IEEE 802.15.4/Zigbee Standard Under Rear Time Constraint", International Journal of Computer Networks \& Communications (IJCNC) Vol.3, No.5, Sep 2011.

[13] Min Zhou, Zhang-Long Nie," Analysis and design of Zigbee MAC layers protocol," International Conference on Future Information Technology and Management Engineering ( FITME), volume 2,p211-215, 2010.

[14] Abhishek Kumar , Eep Gupta, "Study on Zigbee Technology," 3rd International Conference on Electronics Computer Technology, Volume: 6 Pages: 297-301, 2011 